\section{Case Reports in Ophthalmology}

\title{
Combined Acute Haemolytic and Secondary Angle Closure Glaucoma following Spontaneous Intraocular Haemorrhages in a Patient on Warfarin
}

\author{
Walter Andreatta Stavroula Boukouvala Atul Bansal \\ a University Hospitals Coventry and Warwickshire NHS Trust, Coventry, UK
}

\section{Keywords}

Angle closure - Anticoagulants - Haemolytic glaucoma - Macular degeneration -

Suprachoroidal haemorrhage

\begin{abstract}
Background: To report the first described case of combined haemolytic and acute angle closure glaucoma secondary to spontaneous intraocular haemorrhages in a patient on excessive anticoagulation. To the best of our knowledge, this is the first case reported in the literature presenting with raised intraocular pressure due to both mechanisms. Case Description: A 90-year-old woman presented with acute pain and reduction in vision in the left eye. Her intraocular pressure (IOP) was $55 \mathrm{~mm} \mathrm{Hg}$. There were red tinted blood cells in the anterior chamber giving it a reddish hue. The patient was known to have advanced wet macular degeneration. She was taking oral warfarin for atrial fibrillation. Her international normalised ratio (INR) was 7.7. B-scan ultrasound of posterior segment showed vitreous and suprachoroidal haemorrhages. An ultrabiomicroscopic examination confirmed open angles. A diagnosis of haemolytic glaucoma secondary to intraocular haemorrhages was made. The IOP was controlled medically. Warfarin was withdrawn and oral vitamin K therapy was initiated leading to a rapid INR reduction. Three days later, her anterior chamber became progressively shallower causing a secondary acute angle closure which was managed medically. After 2
\end{abstract}




\section{Case Reports in Ophthalmology}

months, the left IOP was well-controlled without any medications and the eye was not inflamed. Her vision in that eye remained perception of light. Conclusion: Patients with suprachoroidal haemorrhages should be closely monitored as they might subsequently develop acute angle closure despite an initially open angle and well-controlled INR and IOP. Excessive anticoagulation needs to be prevented to minimise the risk of sight-threatening complications.

\section{Background}

Spontaneous vitreous and suprachoroidal haemorrhage are very rare complications in patients affected by neovascular age-related macular degeneration (AMD) on excessive anticoagulation. Nevertheless, they are extremely serious. While red blood cells haemolysis can result in uncontrolled intraocular pressure (IOP), suprachoroidal haemorrhages often lead to blindness. To our knowledge, this is the first case where spontaneous vitreous and suprachoroidal haemorrhages led to haemolytic glaucoma first, followed by an acute secondary angle closure attack.

\section{Case Description}

A 90-year-old Caucasian female presented to the eye casualty with a 2-day history of headaches, nausea, and a painful, red left eye with reduced vision.

Past ocular history included stable primary open angle glaucoma, right-eye cataract surgery and bilateral age-related macular degeneration (AMD). The left eye was known to have a disciform scar, amblyopia, and hand movements vision. Her medical history included atrial fibrillation, chronic congestive heart failure, hypertension, hypercholesterolaemia, chronic kidney disease stage 3 , and a previous cerebral vascular accident. She was on oral warfarin, furosemide, ramipril, and simvastatin.

At presentation, the corrected Snellen visual acuity was 6/15 in the right and hand movements in the left eye. The right eye was pseudophakic and had moderate dry AMD. The IOP in the left eye was $55 \mathrm{~mm} \mathrm{Hg}$, the ocular surface was deeply inflamed, the cornea oedematous, and the pupil fixed and mid-dilated.

Nevertheless, the anterior chamber (AC) was moderately deep on van Herick's test (grade 3), showing a red hue secondary to haemolysed red blood cells (Fig. 1). The angles and fundus could not be appropriately assessed due to the oedematous cornea and dense cataract.

The international normalised ratio (INR) was 7.7, while all other haematological and biochemical tests were within normal limits. Ultrasound biomicroscopy (UBM) of the left anterior segment showed normal AC depth and open angles but the ocular ultrasound of the posterior segment revealed a diffuse suprachoroidal and vitreous haemorrhage (Fig. 2). The patient was admitted and required intravenous acetazolamide, topical ocular hypotensive medications, dexamethasone $0.1 \%$, cyclopentolate $1 \%$, and oral glycerol to achieve a IOP of less than $21 \mathrm{~mm} \mathrm{Hg}$. Warfarin was omitted and the patient was given $5 \mathrm{mg}$ of oral vitamin $\mathrm{K}$ which reduced the INR to 1.9. Warfarin was then re-started and the INR kept within the low therapeutic range.

Despite good IOP and INR control, van Herick's grade progressed from 3 to 0 between the third and the sixth day of her hospital stay. Angle closure was confirmed on repeated 
ocular UBM (Fig. 3). Considering the pathophysiology of the angle closure and the visual prognosis, YAG laser peripheral iridotomy was not performed.

A vitreo-retinal opinion was sought, but drainage of the haemorrhage was not considered appropriate due to the risk of recurrent haemorrhage and poor prognosis.

Two months following the acute event, the left eye was comfortable, the corneal oedema had largely resolved, the AC was quiet and returned to its normal depth. Gonioscopy showed open angles and a reddish-brown pigment on the trabecular meshwork. The IOP was wellcontrolled without any ocular hypotensive agents. Despite the poor visual outcome, the patient was pleased that the left eye returned to be comfortable.

\section{Discussion}

We postulate that neovascular AMD predisposed our patient to a macular haemorrhage which progressed to a large vitreous and suprachoroidal bleed because of the high clotting time induced by warfarin. The migration of haemolysed red blood cells into the AC led to a rise in IOP despite the presence of open angles. Subsequently, the mass effect of the suprachoroidal haemorrhage and increasing uveal congestion resulted in secondary angle closure. To our knowledge, the occurrence of both mechanisms in a single patient has never been reported before.

While vitreous haemorrhage can occur in neovascular AMD, haemolytic glaucoma is a rare complication $[1,2]$. The IOP is believed to increase because of the obstruction of the trabecular meshwork caused by haemolysed red blood cells [1]. Haemolytic glaucoma is usually managed conservatively but $\mathrm{AC}$ washout with or without trans pars plana vitrectomy was required in cases with refractory raised IOP $[1,2]$.

In our case, the red hue of the AC directed us towards the correct diagnosis and the IOP responded well to medical treatment. Once the acute episode resolved, gonioscopy excluded rubeosis and other pathologies which can cause raised IOP in the presence of open angles.

Although suprachoroidal haemorrhages most commonly occur intraoperatively, they can rarely be spontaneous in patients treated with thrombolytic agents, low molecular weight heparin and warfarin [3]. Other identified risk factors include hypertension, atherosclerosis, and advanced age.

Only a few case reports described acute angle closure secondary to spontaneous suprachoroidal haemorrhages in AMD patients on warfarin [4-7]. These are usually managed conservatively but YAG laser peripheral iridotomies have been performed. In cases without angle closure, surgical evacuation has also occasionally been attempted [3].

The visual prognosis of these patients is very poor independently from their treatment. This condition can have catastrophic consequences if the affected eye is the better seeing eye as it might be the case in AMD patients. Therefore, the presence of advanced atrophic and neovascular AMD should always be considered in the risk assessment of a patient requiring anticoagulation. Similarly, the need for anticoagulation should be reviewed if a patient develops neovascular AMD as previous cases report the occurrence of spontaneous suprachoroidal haemorrhages in patients with INRs within therapeutic range [5]. 


\section{Conclusion}

To the best of our knowledge, this is the first reported case where vitreous and suprachoroidal haemorrhages led to secondary open angle haemolytic glaucoma and the subsequent anterior movement of the lens-iris diaphragm resulted in secondary acute angle closure.

We propose that patients with acutely raised IOPs on anticoagulants and a poor view of the fundus, irrespectively of the AC depth, should have an urgent ocular ultrasound scan and blood clotting profile. Patients with suprachoroidal haemorrhages should be closely monitored as they might develop acute angle closure despite well-controlled IOP and INR.

Good communication between healthcare practitioners, the patients and their families is essential to ensure the best possible INR monitoring and minimise the risk of these complications.

\section{Statement of Ethics}

Informed consent was obtained.

\section{Disclosure Statement}

No conflicting relationship exists for any author.

\section{References}

Phelps CD, Watzke RC: Hemolytic glaucoma. Am J Ophthalmol 1975;80:690-695.

Singh H, Grand MG: Treatment of blood-induced glaucoma by trans pars plana vitrectomy. Retina 1981;1:255-257.

3 Chu TG, Green RL: Suprachoroidal hemorrhage. Surv Ophthalmol 1999;43:471-486.

4 Chandra A, Barsam A, Hugkulstone C: A spontaneous suprachoroidal haemorrhage: a case report. Cases J 2009;2:185.

5 Knox FA, Johnston PB: Spontaneous suprachoroidal haemorrhage in a patient with age-related macular degeneration on excessive anticoagulation therapy. Eye 2002;16:669-670.

6 Alexandrakis G, Chaudhry NA, Liggett PE, Weitzman M: Spontaneous suprachoroidal hemorrhage in age-related macular degeneration presenting as angle-closure glaucoma. Retina 1998;18:485-486.

7 Lewis H, Sloan SH, Foos RY: Massive intraocular hemorrhage associated with anticoagulation and agerelated macular degeneration. Graefes Arch Clin Exp Ophthalmol 1988;226:59-64. 


\section{Case Reports in Ophthalmology}

\begin{tabular}{l|l}
\hline Case Rep Ophthalmol 2016;7:233-238 \\
\hline DOI: 10.1159/000452440 & $\begin{array}{l}\text { ○ 2016 The Author(s). Published by S. Karger AG, Basel } \\
\text { www.karger.com/cop }\end{array}$ \\
\hline
\end{tabular}

Andreatta et al.: Combined Acute Haemolytic and Secondary Angle Closure Glaucoma following Spontaneous Intraocular Haemorrhages in a Patient on Warfarin

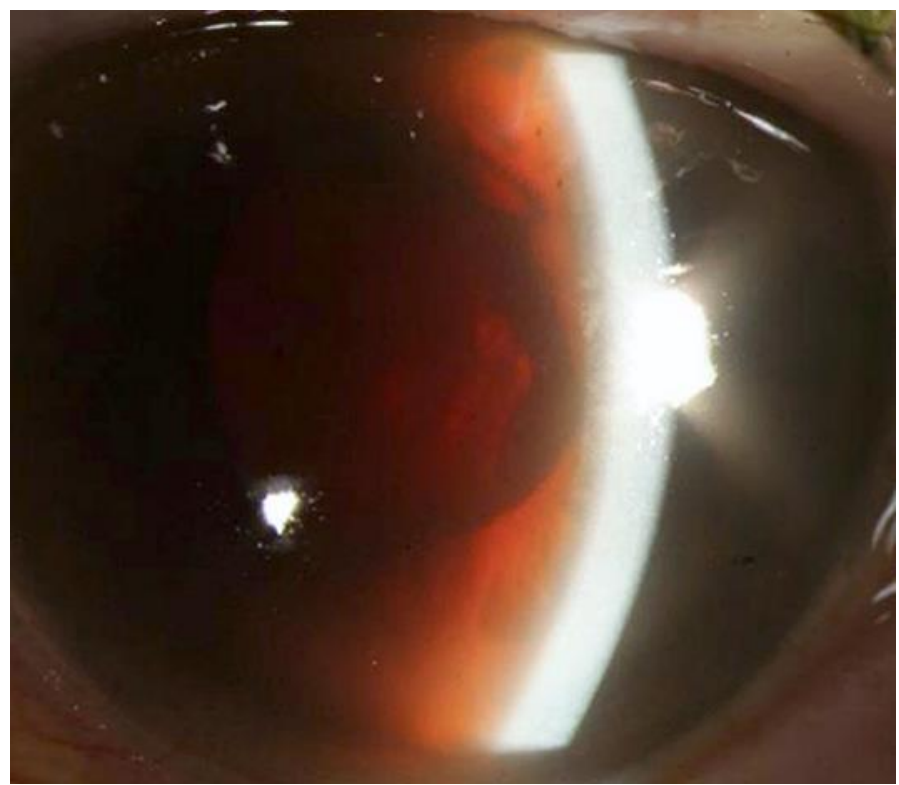

Fig. 1. Red hue of the aqueous due to haemolysed blood cells migrating from the posterior segment.

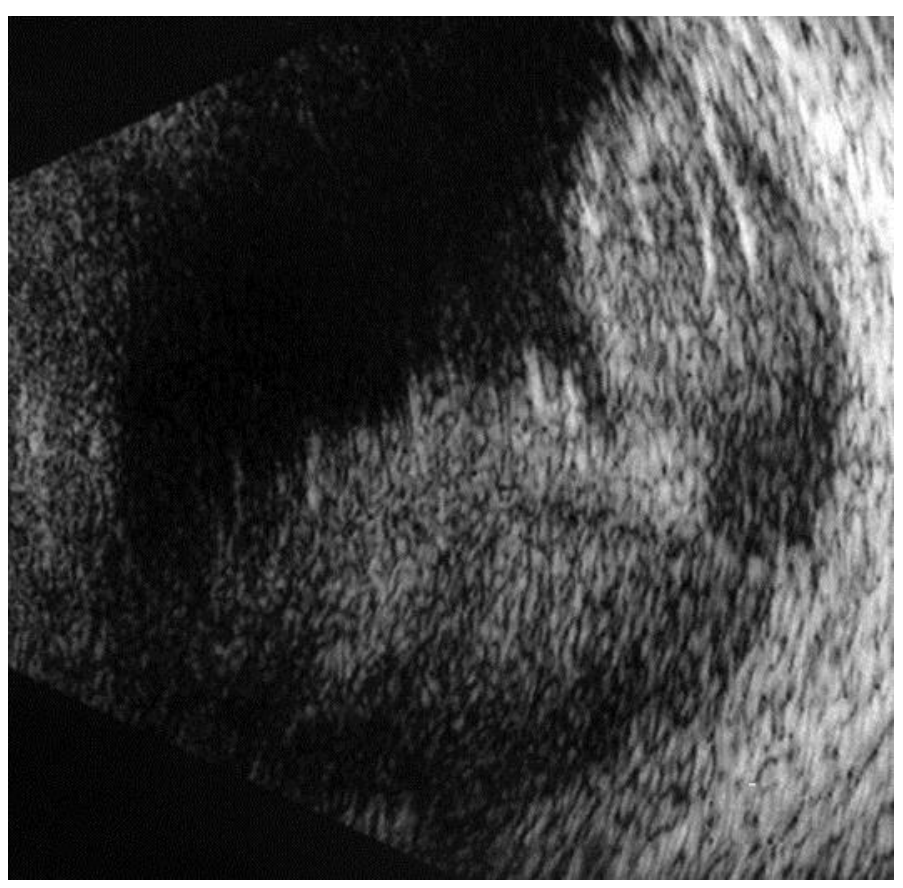

Fig. 2. B-mode ocular ultrasound of the left posterior segment shows an intraocular hyperechoic signal and vitreous opacities. 
Case Reports in
Ophthalmology

Case Rep Ophthalmol 2016;7:233-238

DOI: $10.1159 / 000452440$

(c) 2016 The Author(s). Published by S. Karger AG, Basel www.karger.com/cop

Andreatta et al.: Combined Acute Haemolytic and Secondary Angle Closure Glaucoma following Spontaneous Intraocular Haemorrhages in a Patient on Warfarin

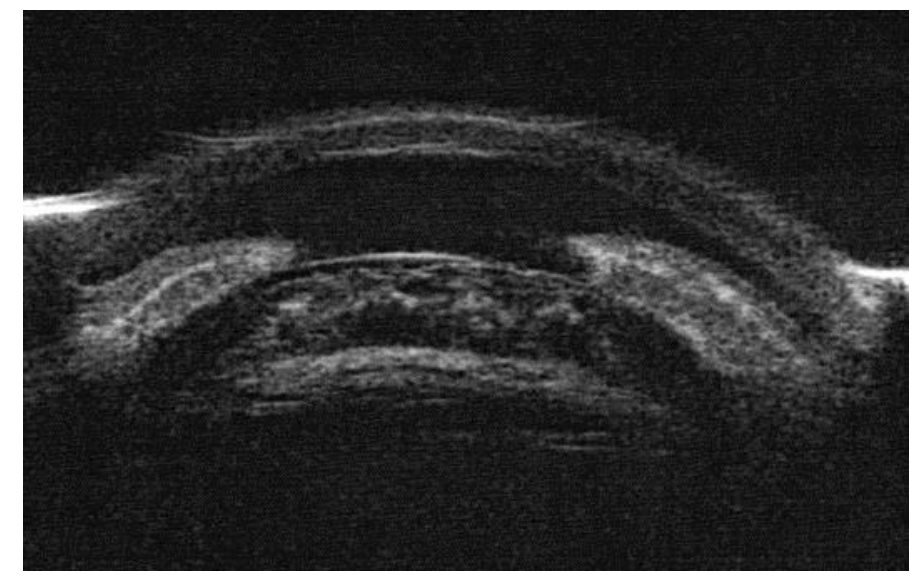

Fig. 3. Left eye anterior segment UBM demonstrates a shallow AC with closed angles. 